\title{
Kelvin-Helmholtz Instabilities Occurring at a Nacelle Exhaust
}

\author{
Julien Manera, Benoît Schiltz, Romain Leneveu, Stéphane Caro, Jonathan Jacqmot \\ Free Field Technologies SA - www.fft.be \\ Axis Park Louvain-La-Neuve,Rue Emile Francqui 1, \\ B-1435 Mont St Guibert, Belgium \\ Sjoerd Rienstra, TUE \\ Technical University of Eindhoven
}

\begin{abstract}
The difference of flow velocity between the free stream and the jet stream at a nacelle exhaust generates a shear layer where some hydrodynamic instabilities, called KelvinHelmholtz instabilities, can occur. Some instabilities occur in the shear layer in the computations with ACTRAN/DGM a time domain code which solves the LEE equations (see, ${ }^{1},{ }^{2}$ and $^{3}$ ) but it is often difficult to conclude whether they are physical or not. This document recalls the theory on the Kelvin-Helmholtz instabilities and gives guidelines to sort out the physical and numerical instabilities. This methodology is put into practice on concrete cases studied in the frame of the TURNEX project.
\end{abstract}

\section{Kelvin-Helmholtz Instabilities}

An incompressible inviscid parallel mean flow with an infinitely thin shear layer dividing two regions with uniform but different velocity profiles, is linearly unstable according to the classic theory of Kelvin and Helmholtz. ${ }^{4,5}$ This remains true for shear layers of finite thickness. ${ }^{6}$ Since it is essentially the concentration of vorticity that makes the flow unstable, a (subsonic) compressible flow is unstable in qualitatively the same way, although now acoustic waves may excite the instability. ${ }^{7-13}$

Due to linearity, a perturbed quantity $q$ can we written as a sum or integral over space-time Fourier modes, of which the most unstable has our interest. Assuming the mean flow being oriented in positive $x$-direction, then $q$ may be given, in the usual complex form, by

$$
q(x, y, z, t)=A(y, z) \mathrm{e}^{\mathrm{i} \omega t-\mathrm{i} k x} .
$$

$\omega$ and $k$ are related by a dispersion relation, so that locally we can say that $\omega=\omega(k)$. The phase and group velocities are given by

$$
v_{p h}=\frac{\omega}{k}, \quad v_{g}=\frac{\mathrm{d} \omega}{\mathrm{d} k} .
$$

The phase velocity is the easily observed speed of the wave tops, while the group velocity is the speed of the wave energy. The mean flow is said to be convected unstable if no instability mode has group velocity equal to zero. ${ }^{14}$ It is said to be absolute unstable if some unstable mode has group velocity equal to zero. Temporal modes are modes for which we take a real value of the wave number $k$ and seek corresponding complex $\omega$ which yields an unstable mode if $\operatorname{Im}(\omega)<0$. Spatial modes are modes for which we take a real value of the frequency $\omega$ and seek corresponding complex $k$ which yields an unstable (rightrunning) mode if $\operatorname{Im}(k)>0$.

\footnotetext{
*Corresponding author: julien.manera@fft.be

Copyright (c) 2008 by the authors. Published by the American Institute of Aeronautics and Astronautics, Inc. with permission.
} 
This last scenario ${ }^{a}$ is typical of the present class of problems, where the instability is acoustically excited by a source of fixed and given real frequency. It appears that a Kelvin-Helmholtz unstable mean flow, with no density gradients opposite to the direction of gravity ${ }^{\mathrm{b}}$, is only convected unstable with spatial modes.

In the numerical problem, however, it is not obvious that there are no other instabilities. It is one of the goals of the present research to confirm that no other instabilities occur than the physical ones.

An important property of spatial modes for shear layers of finite thickness is that they are only unstable (exponentially increasing with $x$ ) for shear layers that are thin enough non-dimensionally. Since compressibility is not relevant, it means thin enough compared to a suitable hydrodynamic wavelength $U / \omega$. This comes down to a small enough Strouhal number. According to Michalke, ${ }^{15,16}$ the relevant Strouhal number is defined as

$$
S_{t}=2 \pi \frac{f_{K H} \theta_{m}}{U_{\text {jet }}-U_{\text {free }}}
$$

where

- $f_{K H}$ is the Kelvin-Helmholtz instabilities frequency. Note that we make an explicit distinction between this and the frequency of the driving source, because in the end we want to show that the frequency of the observed instability is indeed equal to the one of the source.

- $U_{\text {jet }}$ is the velocity of the jet mean flow (in practice, at the jet centre line);

- $U_{\text {free }}$ is the velocity of the free mean flow that surrounds the jet (sufficiently far away from the jet).

- $\theta_{m}$ is the shear layer momentum thickness, defined as:

$$
\theta_{m}=\int_{0}^{\infty}\left\{\frac{1}{4}-\left(\frac{U(r)-\bar{U}}{\Delta U}\right)^{2}\right\} \mathrm{d} r .
$$

$\bar{U}=\frac{1}{2}\left(U_{\text {jet }}+U_{\text {free }}\right)$ is the mean flow velocity halfway the shear layer and $\Delta U=U_{\text {free }}-U_{\text {jet }}$ is the difference between free stream and jet stream velocities. If the density varies between the two domains, the momentum density $\rho U$ should be taken into account instead of the velocity $U$, but this does not make any difference for the order of magnitude of the effect.

Michalke's results are illustrated in Figure 1 for various situations.
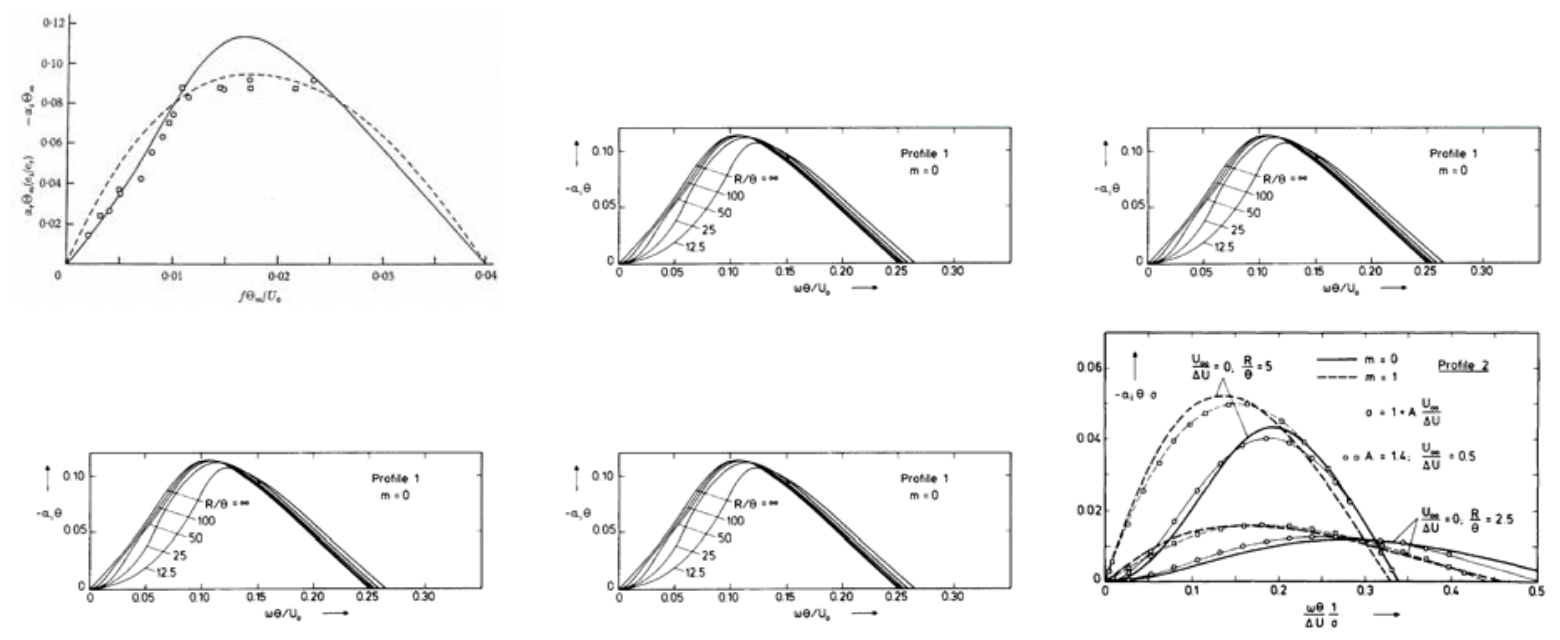

Figure 1. Examples of Michalke's results for 2D (upper-left) and 3D cylindrical jet flow. Note that $k=\alpha$.

We note the following observations.

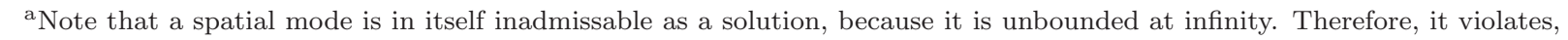
with any nonzero amplitude, the assumption of uniform smallness that is required for linearisation. Nevertheless, it is believed that a spatial mode is a useful solution anywhere it is locally small.

blike a cup of tea held upside down 
- When the instability wavelength and the shear layer thickness are much smaller than the jet diameter, the instability propagation velocity should be nearly equal to the average of inner and outer flow velocities. This is clear from elementary symmetry arguments.

- When the instability wavelength is larger than the jet diameter, the instability propagation velocity should be nearly the same as the one of the jet flow. This is less obvious; the symmetry is broken while the jet and the instability wave tend to coincide.

- The Kelvin-Helmholtz instabilities are only driven by the acoustic source (i.e., their frequency is equal to the acoustic frequency). This is an obvious result from the linearisation combined with arguments of causality and an undisturbed initial flow.

Michalke's results suggests a criterion that any shear layer instability turns into stable, decaying perturbations when the above Strouhal number $S_{t}$ based on the shear layer momentum thickness (Equation 3) is larger than a certain threshold value,

$$
S_{t}>S_{c}
$$

which is broadly speaking of order unity, but depends on further details of the geometry and type of perturbation $\left(\operatorname{see}^{15}\right.$ and $\left.{ }^{16}\right)$.

It explains why any infinitely thin shear layer (the vortex sheet model) is always Kelvin-Helmholtz unstable: the Strouhal number is always zero, for whatever frequency.

This reveals a weakness of the vortex sheet jet models. On the one hand these models are extremely useful, because their relatively simple geometry allows a deep analysis of the physics. Especially the celebtrated solution for a circular jet with co-flow by Munt ${ }^{10}$ has shown to be seminal, in particular in the Turnex project. ${ }^{17-19}$ On the other hand, one aspect which is not unimportant in more realistic cases is not included: the stabilizing effect of a finite shear layer thickness. ${ }^{20}$

\section{Application: a Munt Type Problem}

The theory explained in Section I is put into practice to the model described in Section II.A and in. ${ }^{3}$ Figure 2 shows the mode of order $(9,1)$ at $866 \mathrm{~Hz}$, where instabilities occur in the shear layer.

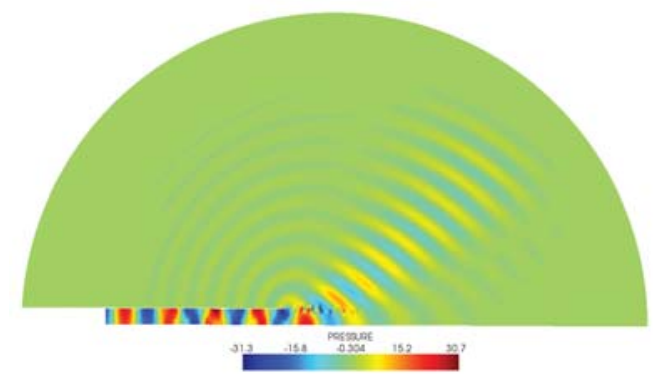

Figure 2. Case 1a, Approach, Mode (9,1), physical pressure distribution

\section{II.A. Model Description}

The case 1a of the TURNEX WP2 is studied here at approach conditions: the geometry corresponds to an extended Munt solution (annular duct), where the flow velocity in the bypass stream and in the free stream are theoretically uniform. They are respectively set to $155 \mathrm{~m} . \mathrm{s}^{-1}$ and $74.5 \mathrm{~m} . \mathrm{s}^{-1}$ ( $\mathrm{see}^{3}$ ). The center-body is acoustically hard and extends indefinitely downstream of the bypass exit plane (Figure 3).

The finite element model is axisymmetric, and the flow, density and pressure profiles are computed with a Matlab routine. As suggested by ${ }^{20}$ and, ${ }^{3}$ the shear layer has a conic shape (the opening angle is set to $10^{\circ}$ ), where the flow velocity varies linearly between the free stream and the jet stream (Figure 4). 


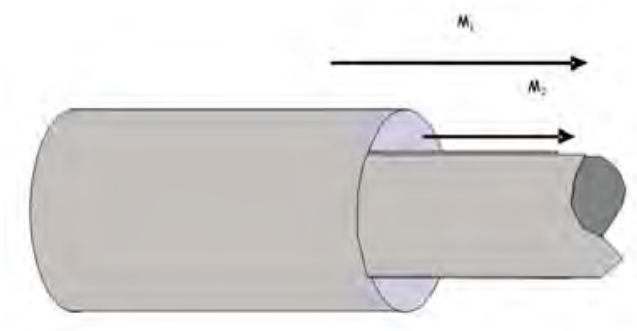

Figure 3. Case 1a, geometry
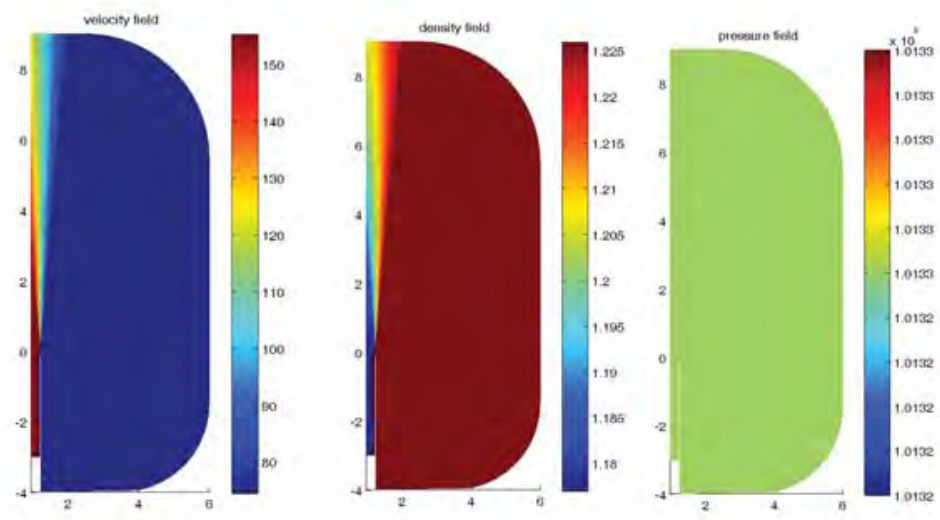

Figure 4. Case 1a, velocity, density and pressure fields at approach

The mesh is presented on Figure 5, where some field points (i.e., virtual microphones) are placed in the continuation of the duct. They allow to plot the pressure variation at different time steps inside the shear layer. The model is excited with the mode $(9,1)$.

\section{II.B. Instability Phase Speed}

The pressure ${ }^{\mathrm{C}}$ inside the shear layer is plotted on Figure 6 distribution at different time steps. This plot allows to compute the phase speed of the instability. This appears to be equal to the average flow velocity between inner and outer flows (i.e., $115 \mathrm{~m} . \mathrm{s}^{-1}$, see (Equation 6).

$$
V=\frac{0.045}{0.023375-0.0229856} \approx 115 \mathrm{~m} \cdot \mathrm{s}^{-1}
$$

\section{II.C. Instability Wavelength}

The pressure distribution inside the shear layer is plotted in Figure 7 at $t=0.023375 \mathrm{~s}$. The wavelength of the instability can be measured from this plot and is equal to $0.125 \mathrm{~m}$. (Equation 7). This leads to an instability frequency equal to $924 \mathrm{~Hz}$ (Equation 8), which is in reasonable agreement with the excitation frequency of $866 \mathrm{~Hz}$.

$$
\lambda=0.535-0.41=0.125 \mathrm{~m}
$$

${ }^{\mathrm{c}}$ This corresponds to the real or imaginary part of the pressure (with a suitably chosen complex phase) in the usual complex representation in case of time harmonic perturbations. 


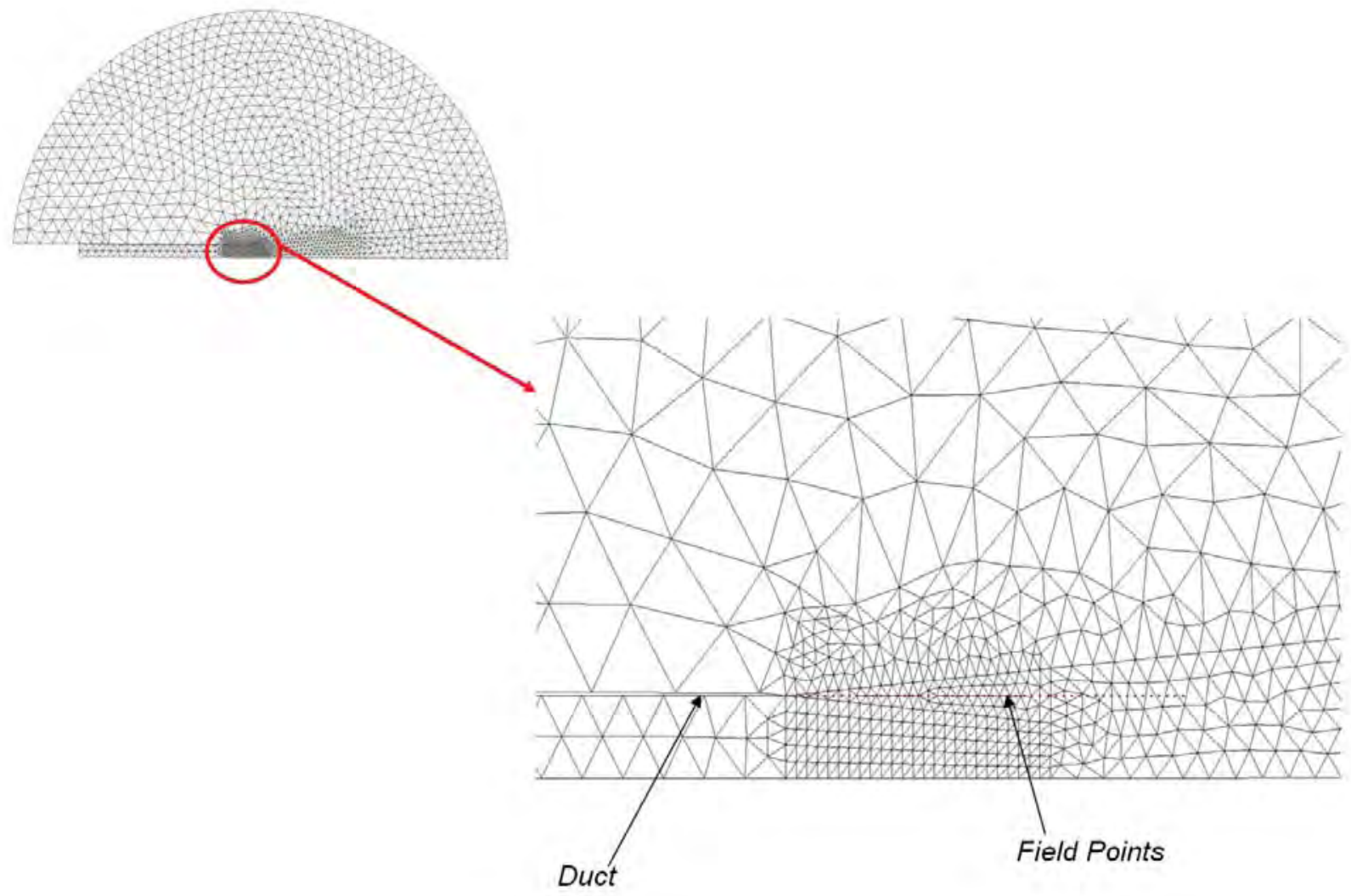

Figure 5. Case 1a, Mesh and virtual microphones location

5 of 18 

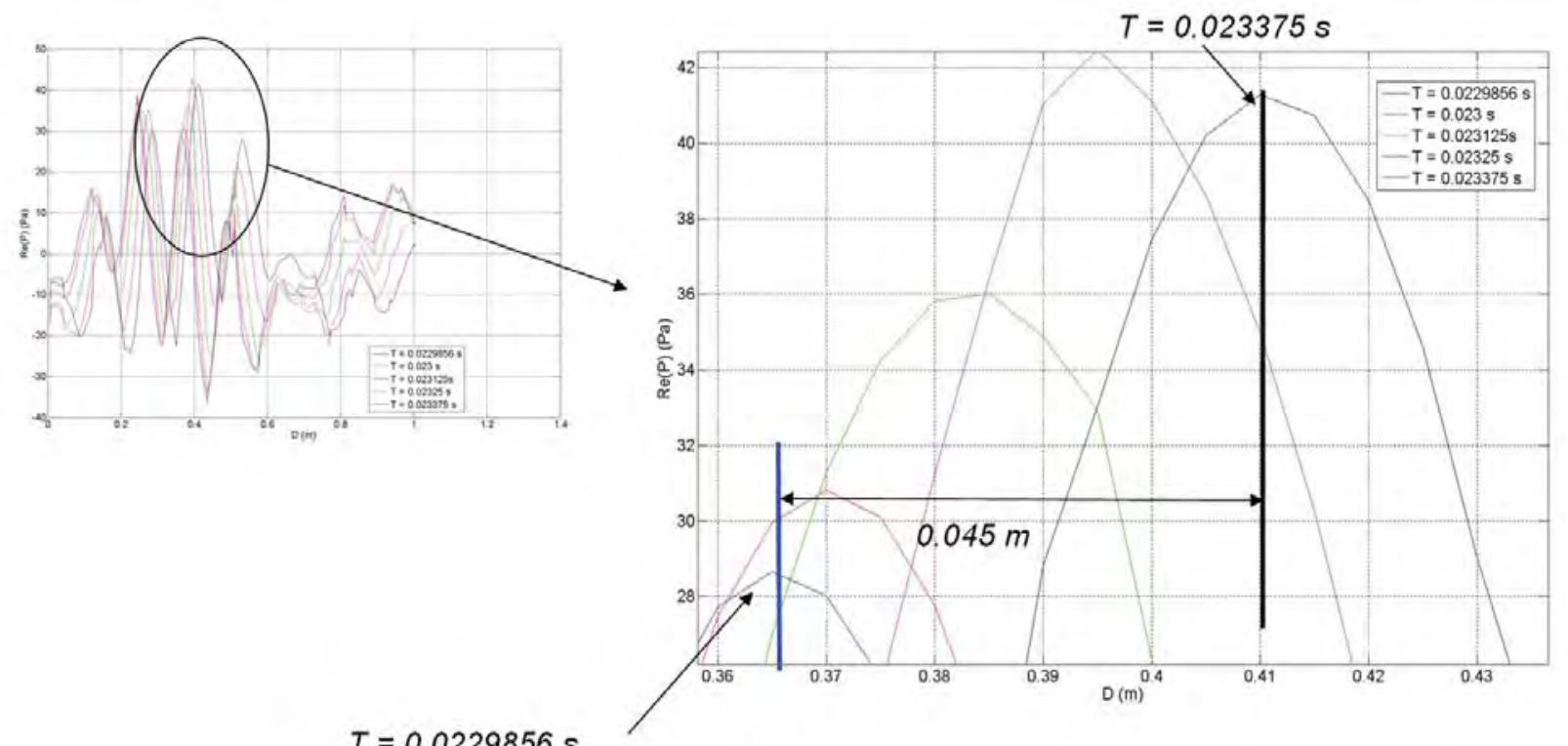

$T=0.0229856 \mathrm{~s}$

Figure 6. Physical pressure distribution inside the shear layer at different time steps; computation of the instability phase speed

$$
f_{K H}=\frac{V}{\lambda}=\frac{115.56}{0.125} \approx 924 \mathrm{~Hz}
$$

\section{II.D. Michalke Criterion}

The Michalke criterion (Equation 3) can be computed because the momentum thickness (Equation4) is reduced in this particular case. This is due to:

- The weak variation of the density. This allows to take into account the velocity in the momentum thickness computation instead of the momentum density;

- The conic shape of the shear layer, which reduces considerably the original formula.

The new expression of the momentum thickness is shown in Equation 9.

$$
\theta_{m}=\int_{0}^{\delta}\left\{\frac{1}{4}-\left(\frac{U_{\text {jet }}+\frac{r}{\delta} \Delta U-\bar{U}}{\Delta U}\right)^{2}\right\} \mathrm{d} r=\frac{\delta}{6}=\frac{2 L \tan \frac{\alpha}{2}}{6}
$$

Where (see also Figure 8):

- $L$ is the distance after which the Kelvin-Helmholtz instabilities should stop growing;

- $\alpha$ is the opening angle of the shear layer;

- $\delta$ is the shear layer thickness.

Therefore, the Michalke criterion for this particular case says that the Strouhal number $S_{t}$ must be larger than 0.9 (Equation 10), as the distance $L$ after which the Kelvin-Helmholtz instabilities stop growing is equal to $0.4 \mathrm{~m}$ (Figure 9 ). The computations parameters are reminded hereafter:

- The instabilities frequency $f_{K H}$ is equal to $924 \mathrm{~Hz}$;

- The free stream flow velocity $U_{\text {free }}$ is equal to $74.5 \mathrm{~m} . \mathrm{s}^{-1}$;

- The jet stream flow velocity $U_{\text {jet }}$ is equal to $155 \mathrm{~m} \cdot \mathrm{s}^{-1}$; 


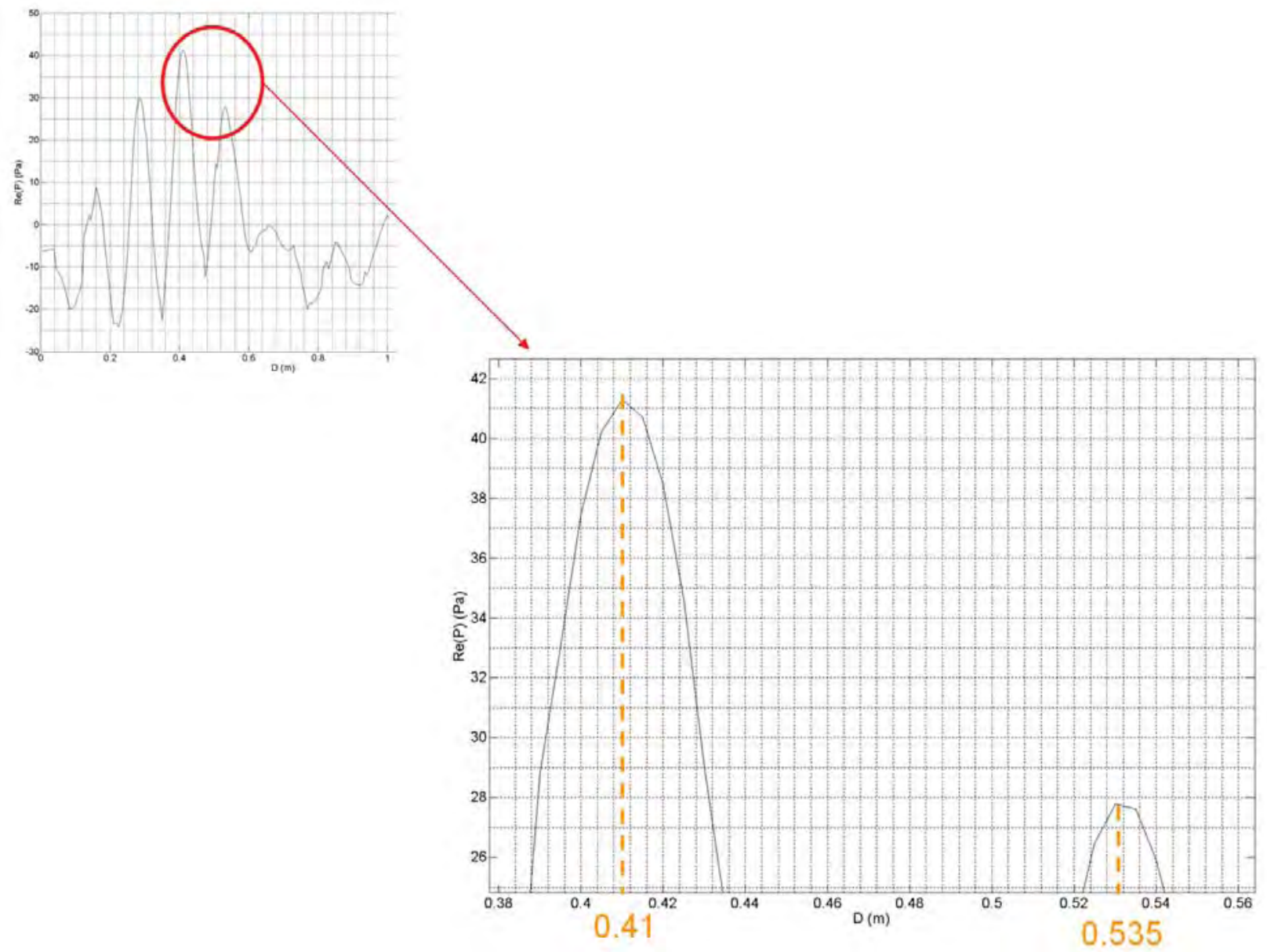

Figure 7. Physical ("real part of") pressure distribution at $t=0.023375$ s. Wavelength Computation

- The opening angle of the shear layer is set to $10^{\circ}$.

$$
S_{t}=2 \pi \frac{f_{K H} \theta_{m}}{U_{\text {jet }}-U_{\text {free }}}=2 \pi \frac{f_{K H} \frac{2 L \tan \frac{\alpha}{2}}{6}}{U_{\text {jet }}-U_{\text {free }}}=0.9
$$

\section{II.E. Conclusions}

Two conclusions can be drawn:

- With the wavelength of the instabilities being smaller than the diameter of the jet, it is normal that they propagate at the average flow velocity between inner and outer flows. This is consistent with the theory (see Section I);

- The instability frequency $(924 \mathrm{~Hz})$ is nearly the same as the source frequency $(866 \mathrm{~Hz})$. Therefore, the instabilities are physical (i.e., these are Kelvin-Helmholtz instabilities, see Section I).

\section{Application: Realistic Problem}

\section{III.A. Model Description}

In Section II.D, the Michalke criterion was computed thanks to the simple shape of the shear layer. In the case of a realistic mean flow, it is not possible to reduce the computation of the momentum thickness to an 


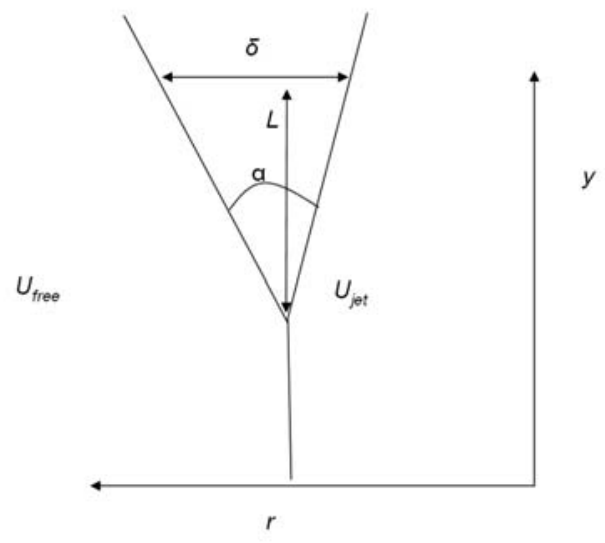

Figure 8. Conic shear layer scheme
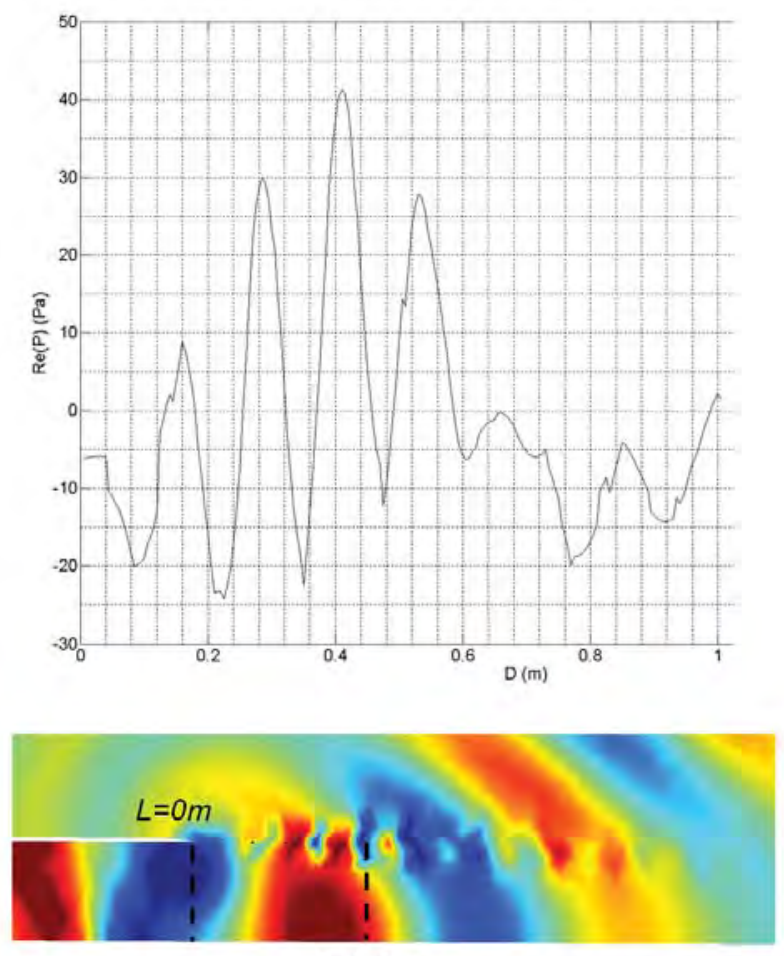

$L=0.4 m$

Figure 9. Pressure map and real part of the pressure inside the shear layer at $t=0.023375 \mathbf{s}$

analytical formula as done in Equation 9: the shear layer shape is often too complex. The TURNEX case 5 is studied in this part as an example of realistic case. The flow and the mesh are shown on Figure 10 and 11 (static-approach flight condition).

Figure 12 shows a pressure map of the plane wave mode at $3000 \mathrm{~Hz}$. Instabilities occur mainly in the external shear layer (between the by-pass flow and the external flow). Figure 13 details the instabilities and plots the direction of acoustic velocity. 


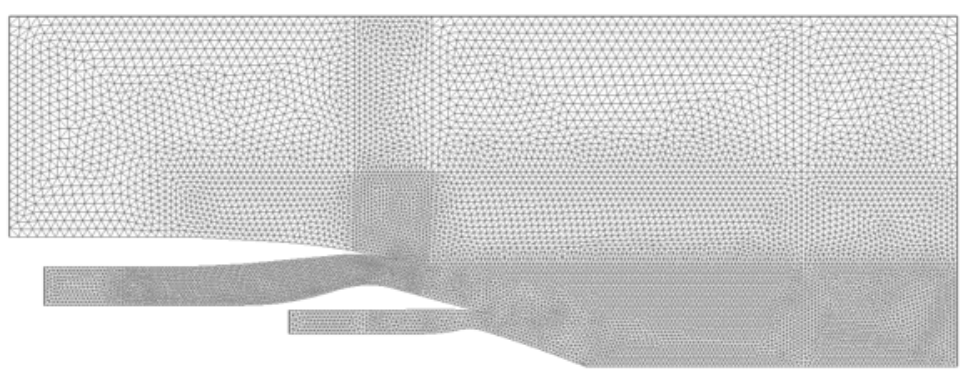

Figure 10. Case 5, mesh

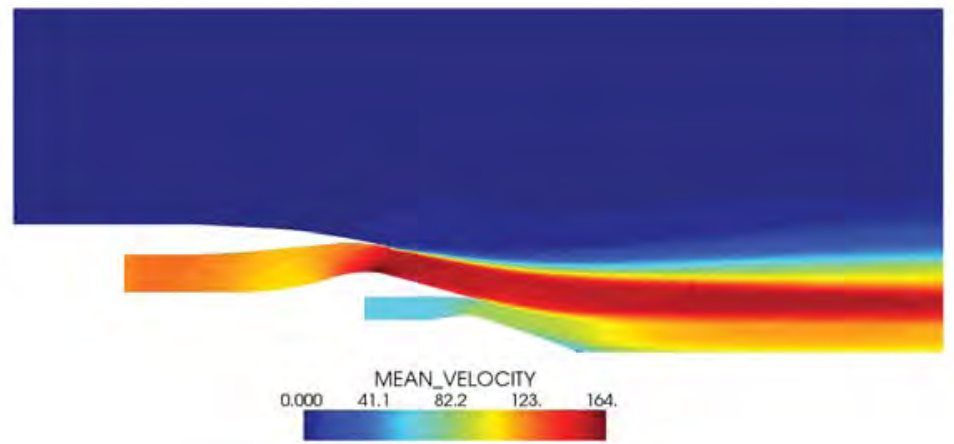

Figure 11. Case 5, interpolated flow on acoustic mesh (colorbar in $[\mathrm{m} / \mathrm{s}]$ )

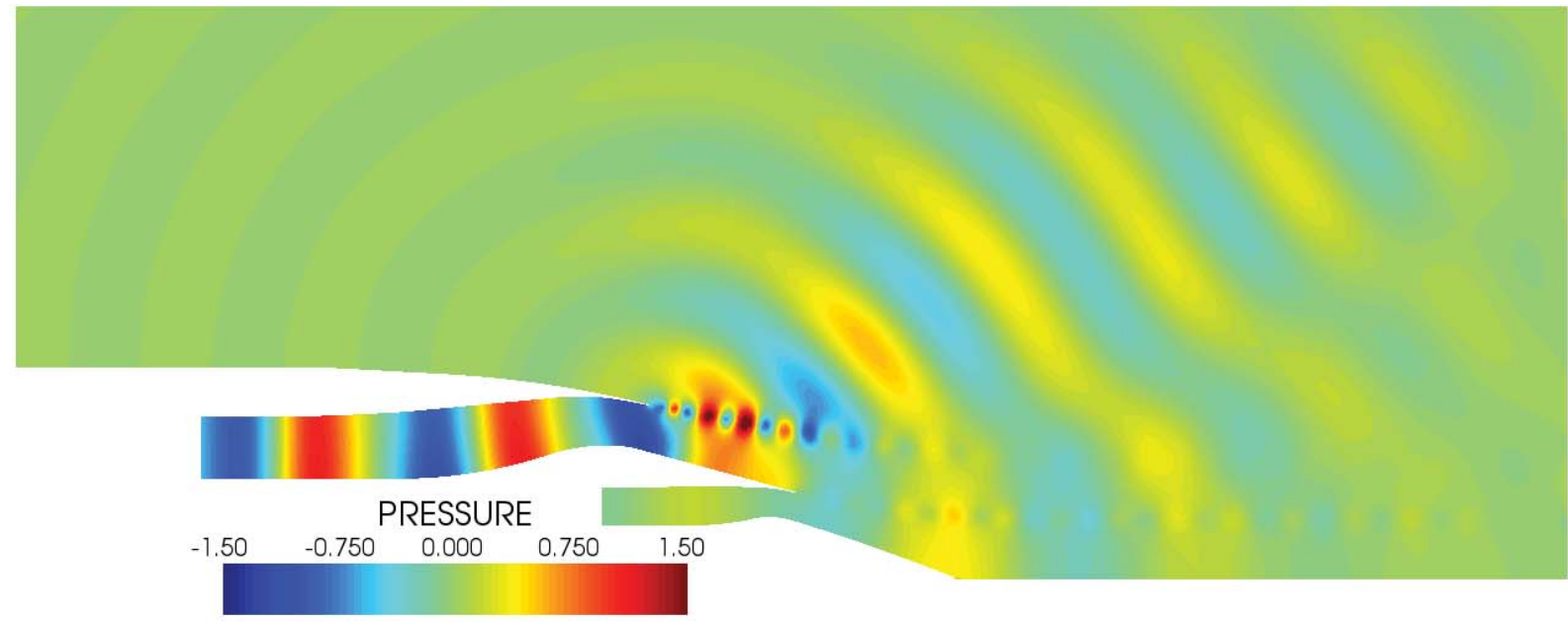

Figure 12. Case 5, pressure map of plane wave mode (excitation frequency : $3000 \mathrm{~Hz}$ )

\section{III.B. Instabilities Wavelength and Velocity Propagation}

The methodology described in Section II.B and II.C is applied to this case in order to compute the instabilities wavelength and their propagation velocity. 


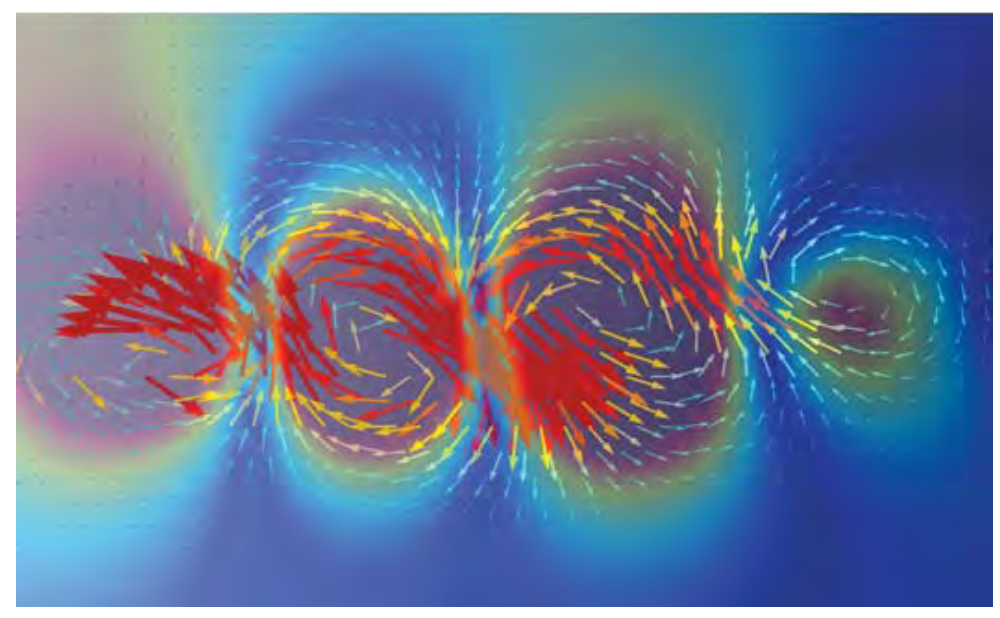

Figure 13. Details of the K-H instabilities with the direction of acoustic velocity

The pressure inside the shear layer is plotted on Figure 14 at different time steps. The distance travelled by a peak can be computed thanks to virtual microphones located inside the shear layer for different time steps. This allows to compute the instabilities phase speed (see Equation 11) which is almost equal to the average flow velocity between by-pass and outer flows (i.e., $\left.75 \mathrm{~m} . \mathrm{s}^{-1}\right)$.

$$
V=\frac{0.058}{0.022150-0.022084} \approx 87.9 \mathrm{~m} \cdot \mathrm{s}^{-1}
$$

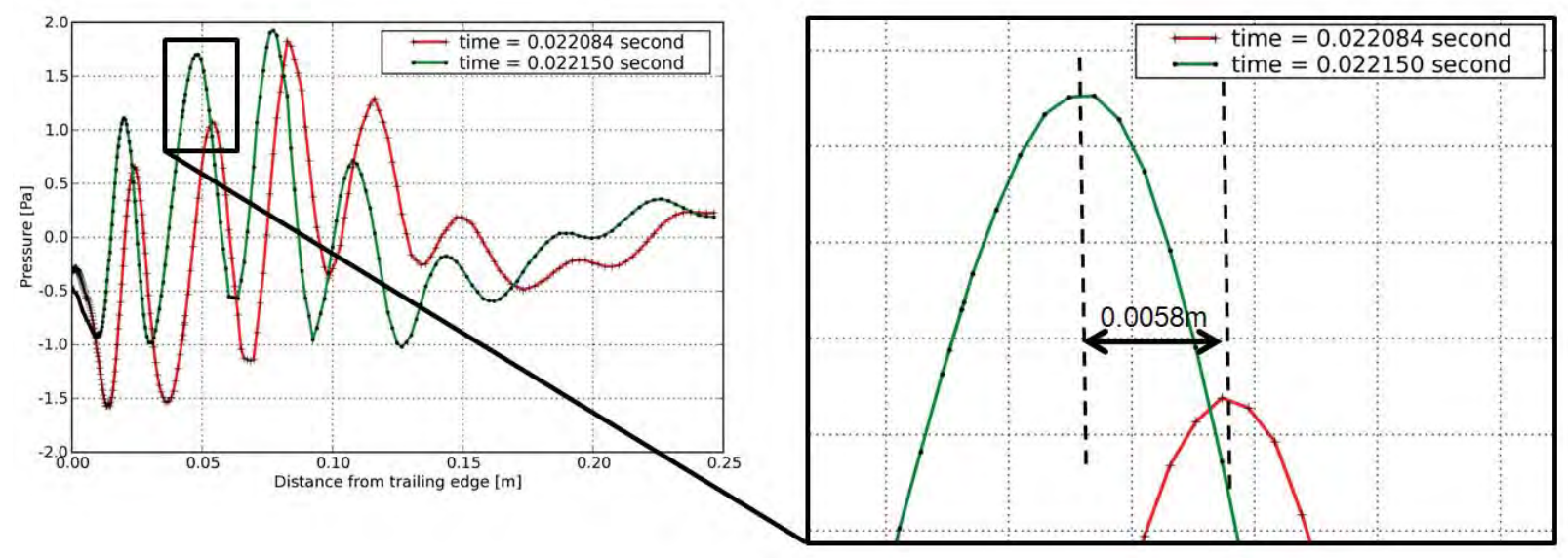

Figure 14. Physical pressure distribution inside the shear layer at different time steps; computation of the instability phase speed

The virtual microphones also allow computing the instability wavelength ( $\lambda=0.029 \mathrm{~m}$ see Figure 15$)$. This leads to an instability frequency equal to $3031 \mathrm{~Hz}$ (Equation 12), which is in agreement with the excitation frequency of $3000 \mathrm{~Hz}$.

$$
f_{K H}=\frac{V}{\lambda}=\frac{87.9}{0.029} \approx 3031 \mathrm{~Hz}
$$

\section{III.C. Michalke Criterion}

The most problematic step to compute the Michalke Criterion is to assess the momentum thickness. The aim of this section is to describe the numerical method used to compute the momentum thickness of realistic 


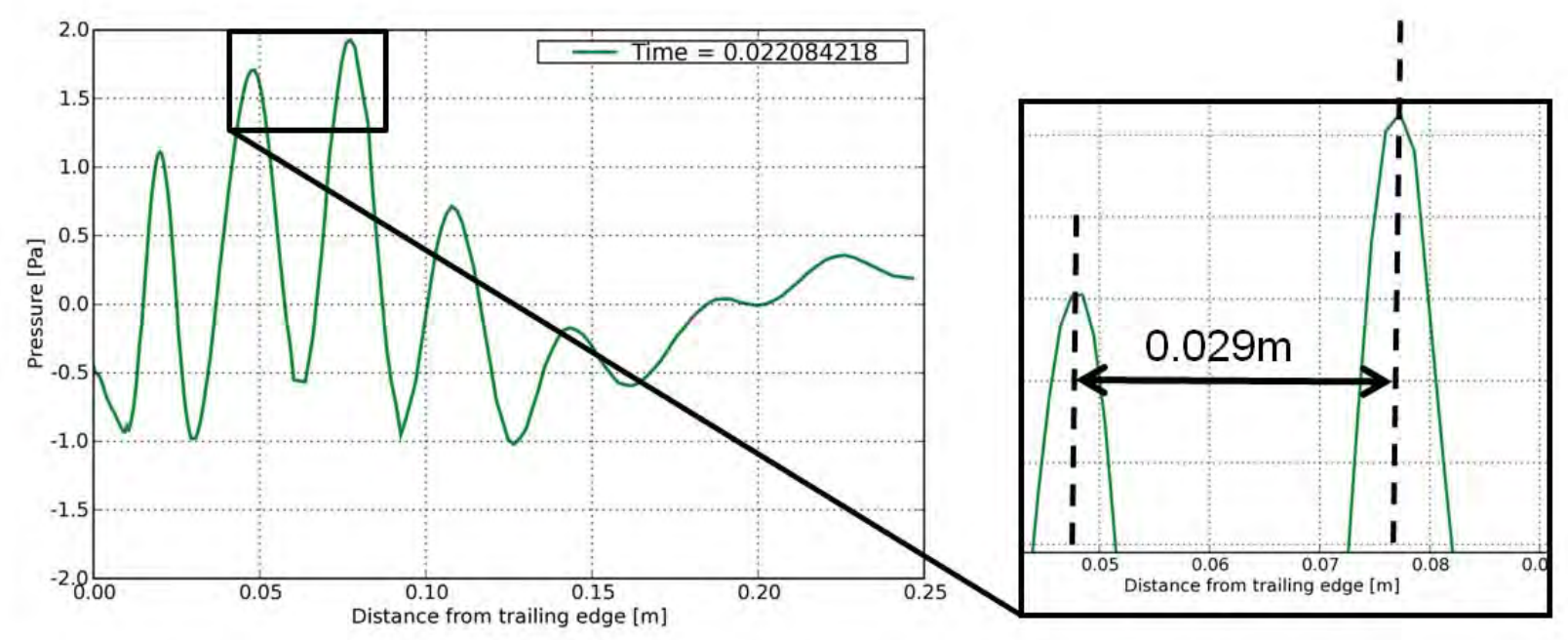

Figure 15. Physical ("real part of") pressure distribution at $t=0.023375$ s. Wavelength Computation

shear layers.

\section{III.C.1. Methodology}

The method consists in interpolating the mean flow on an interpolation mesh (Figure 16).

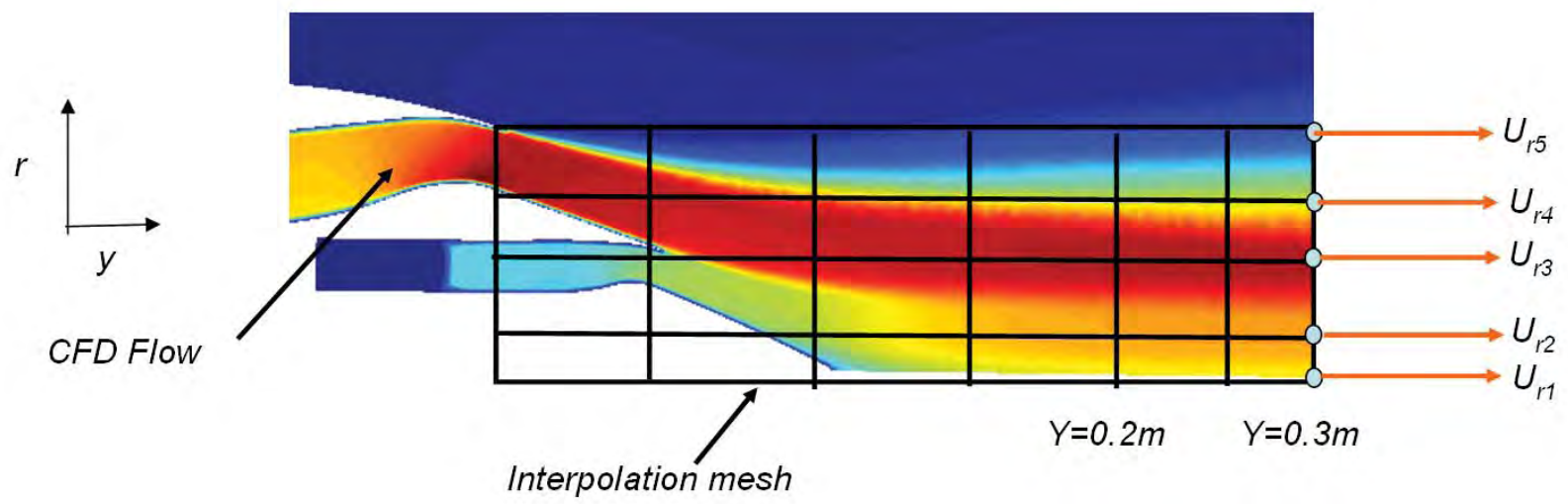

Figure 16. Method for computing the momentum thickness of a CFD mean flow

Thanks to this interpolation sequence, the velocity information is available on every node and can thus be extracted to compute the momentum thickness in different planes. For example, in the $Y=0.3 \mathrm{~m}$ plane, the momentum thickness formula can be reduced to Equation 13.

$$
\theta_{m}=\sum_{i=1}^{5}\left\{\frac{1}{4}-\left(\frac{U_{r i}-\bar{U}}{\Delta U}\right)^{2}\right\} d r
$$

Where $d r$ is the distance between two nodes. 


\section{III.C.2. Validation}

The purpose of this part is to compare the two computational methods of the momentum thickness (Equation 9 and Equation 13) on the case described in Section II.A (i.e., the conic shear layer). This comparison allows to validate the method described in Subsection III.C.1. The momentum thickness is computed in the $Y=1 \mathrm{~m}$ plane. Figure 17 shows the interpolation mesh.

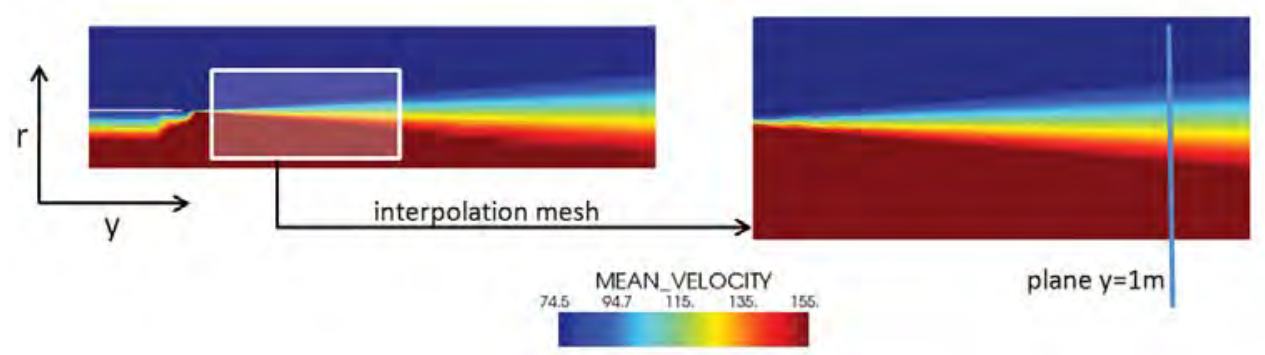

Figure 17. Case 1a, Interpolation mesh (colorbar in $[\mathrm{m} / \mathrm{s}]$ ).

The flow value on each node of the $Y=1 \mathrm{~m}$ plane is known thanks to the flow interpolation. Therefore, it is possible to plot the flow profile (Figure 18) which shows that the shear layer is bordered between $r=1 \mathrm{~m}$ and $r=1.4 \mathrm{~m}$. Equation 13 must be therefore applied in this range (Equation 14).

$$
\theta_{m}=\sum_{r=1}^{1.4}\left\{\frac{1}{4}-\left(\frac{U_{r}-\bar{U}}{\Delta U}\right)^{2}\right\} d r=0.02916 \mathrm{~m}
$$

Where:

- The distance between two nodes $d r$ is equal to $5 \cdot 10^{-3} \mathrm{~m}$;

- The jet stream flow velocity $U_{\text {jet }}$ is equal to $155 \cdot 1 \mathrm{~m} . \mathrm{s}^{-1}$;

- The free stream flow velocity $U_{\text {free }}$ is equal to $74.5 \mathrm{~m} . \mathrm{s}^{-1}$;

- $\bar{U}=\frac{U_{\text {jet }}+U_{\text {free }}}{2}$;

- $\Delta U=U_{\text {free }}-U_{\text {jet }}$.

The comparison between the integral formulation of the momentum thickness for a conic shear layer (Equation 15) and the numerical way (Equation 14) validates this method.

$$
\theta_{m}=\int_{0}^{\delta}\left\{\frac{1}{4}-\left(\frac{U_{j e t}+\frac{r}{\delta} \Delta U-\bar{U}}{\Delta U}\right)^{2}\right\} \mathrm{d} r=\frac{\delta}{6}=\frac{2 L \tan \frac{\alpha}{2}}{6}=0.02918 \mathrm{~m}
$$

\section{III.C.3. Application}

The method of Subsection III.C.1 being validated, it is applied to the TURNEX case 5 at static-approach flow condition described at the beginning of this chapter. The interpolation mesh shown on Figure 19 allows to plot the flow profile in the $Y=0.11 \mathrm{~m}$ plane (Figure 20).

Only the first shear layer is studied in this document. However, the same methodology can be used to determine the Michalke criterion of the second shear layer located between the by-pass flow and the core flow. 


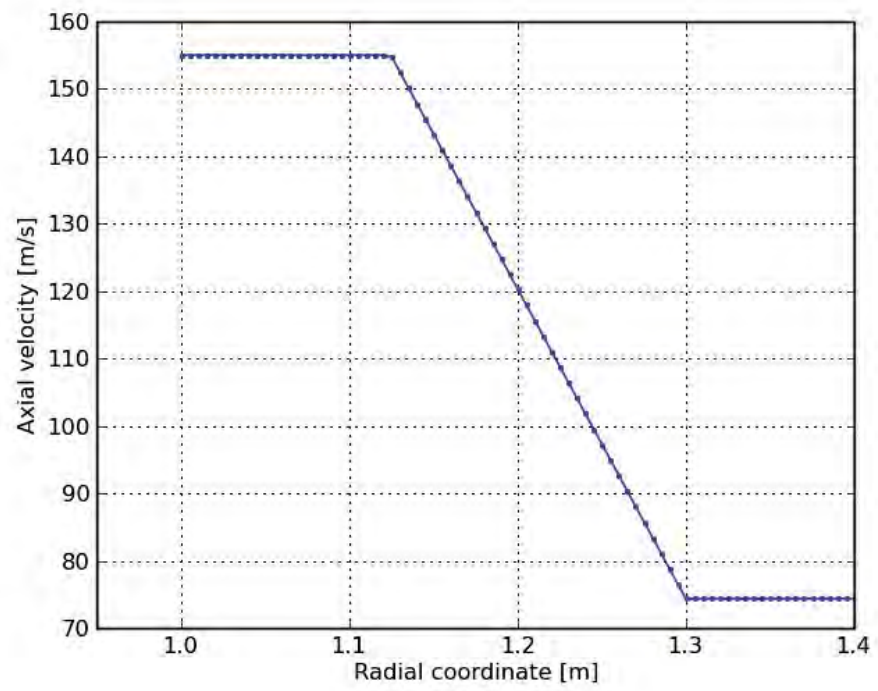

Figure 18. Case 1a, Flow profile in the $Y=1 \mathrm{~m}$ plane

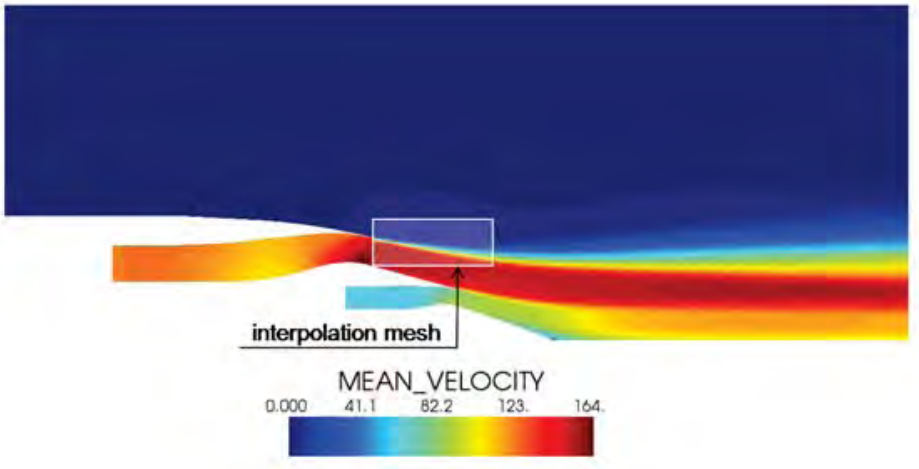

Figure 19. Case 5, Interpolation mesh (colorbar in $[\mathrm{m} / \mathrm{s}]$ ) 


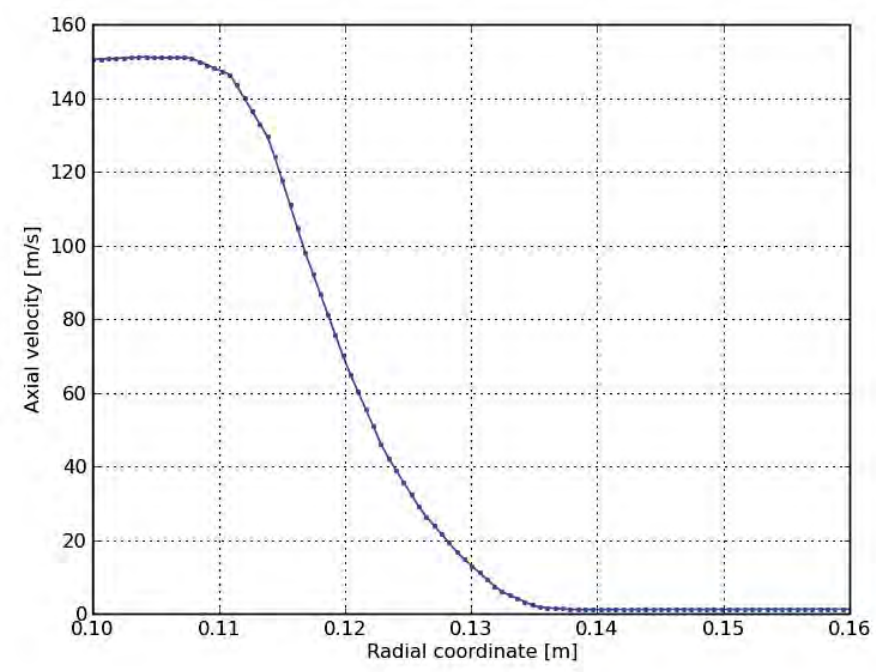

Figure 20. Case 5. Flow profile in the $Y=0.11 \mathrm{~m}$ plane.

The momentum thickness of the first shear layer can be computed thanks to Equation 15 for every Y plane. For the plane $Y=0.11 \mathrm{~m}$, the first shear layer is bordered between $\mathrm{r}=0.10 \mathrm{~m}$ and $\mathrm{r}=0.16 \mathrm{~m}$ (Figure 20). The momentum thickness is therefore equal to $3 \cdot 347 \cdot 10^{-3} \mathrm{~m}$ (Equation 16).

$$
\theta_{m}=\sum_{r=0.10}^{0.16}\left\{\frac{1}{4}-\left(\frac{U_{r}-\bar{U}}{\Delta U}\right)^{2}\right\} d r=3.34 .10^{-3} \mathrm{~m}
$$

Where:

- The distance between two nodes $d r$ is equal to $5.0^{-3} \mathrm{~m}$;

- The by-pass jet stream flow velocity $U_{\text {jet1 }}$ is equal to $151 \mathrm{~m} \cdot \mathrm{s}^{-1}$;

- The exterior jet stream flow velocity $U_{\text {jet2 }}$ is equal to $0 \mathrm{~m} \cdot \mathrm{s}^{-1}$ (static flight condition);

- $\bar{U}=\frac{U_{\mathrm{jet} 1}+U_{\mathrm{jet} 2}}{2}$;

- $\Delta U=U_{\text {jet2 }}-U_{\text {jet1 }}$.

With the momentum thickness being computed for every Y plane the Strouhal number can be computed (Equation 3). Figure 21 illustrates the variation of the Strouhal number with respect to the distance from the trailing edge of the by-pass duct. The amplitude of the instabilities decreases from a distance larger than $0.75 \mathrm{~m}$ (Figure 22). Thanks to the plot shown on Figure 21 the Michalke criterion can be assessed and is equal to 0.33 in this case. This means that the shear layer instabilities become stable once the Strouhal number is larger than 0.33 .

The Strouhal number is plotted for $3000 \mathrm{~Hz}$ and $7000 \mathrm{~Hz}$ on Figure 23. The Michalke criterion at $7000 \mathrm{~Hz}$ is always larger than 0.33 . This means that the instabilities should decrease immediately after their appearance: this is confirmed on the pressure map of Figure 24. 


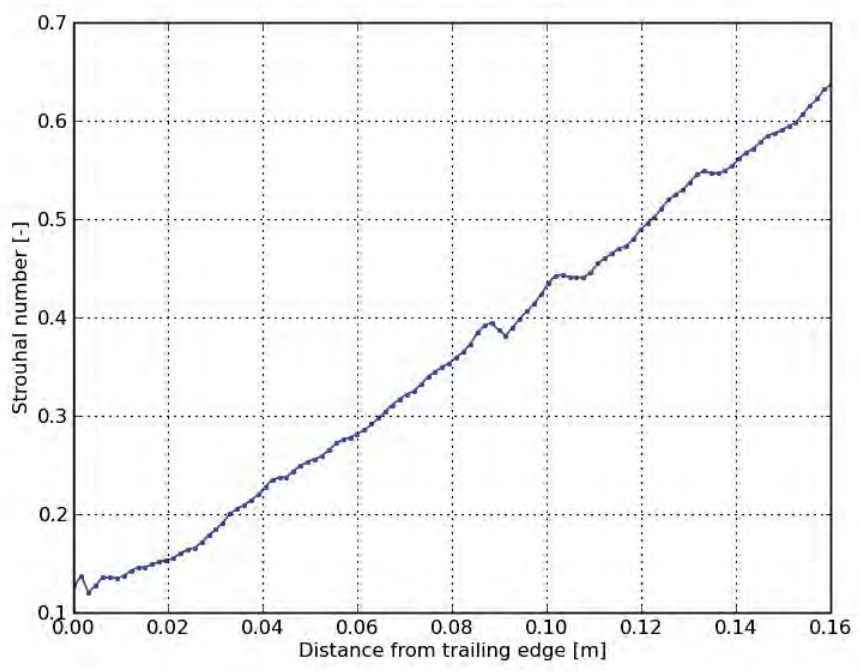

Figure 21. Case 5. Variation of the Strouhal number with the distance from trailing edge.

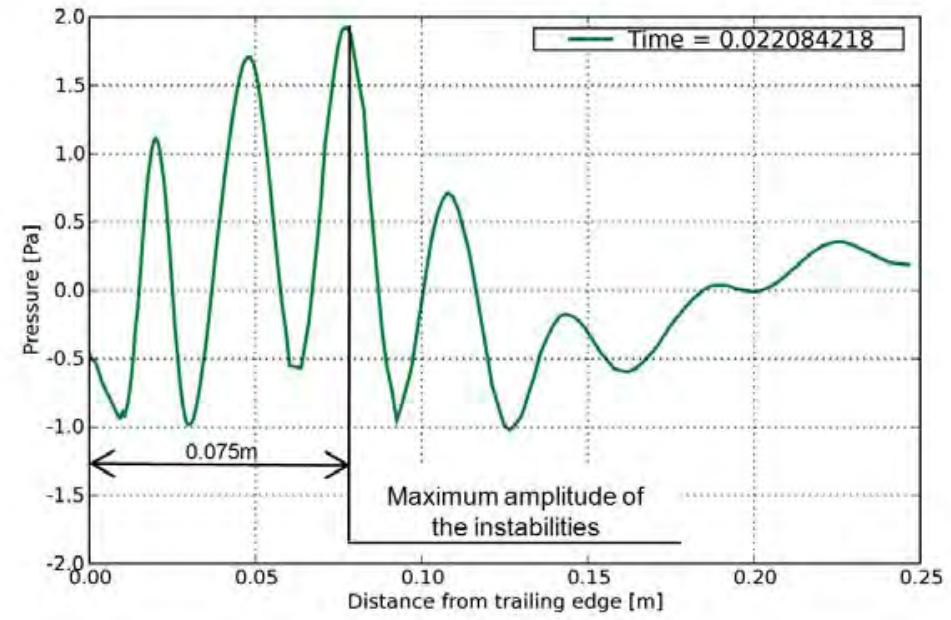

Figure 22. Case 5. location of the maximum of instabilities amplitude. 


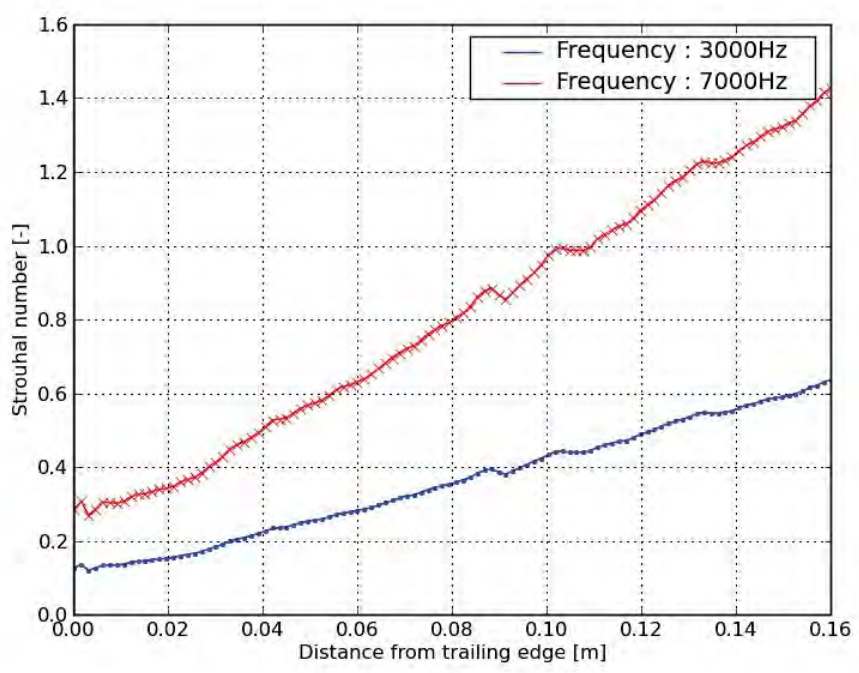

Figure 23. Strouhal number of case 5 for $3000 \mathrm{~Hz}$ and $7000 \mathrm{~Hz}$

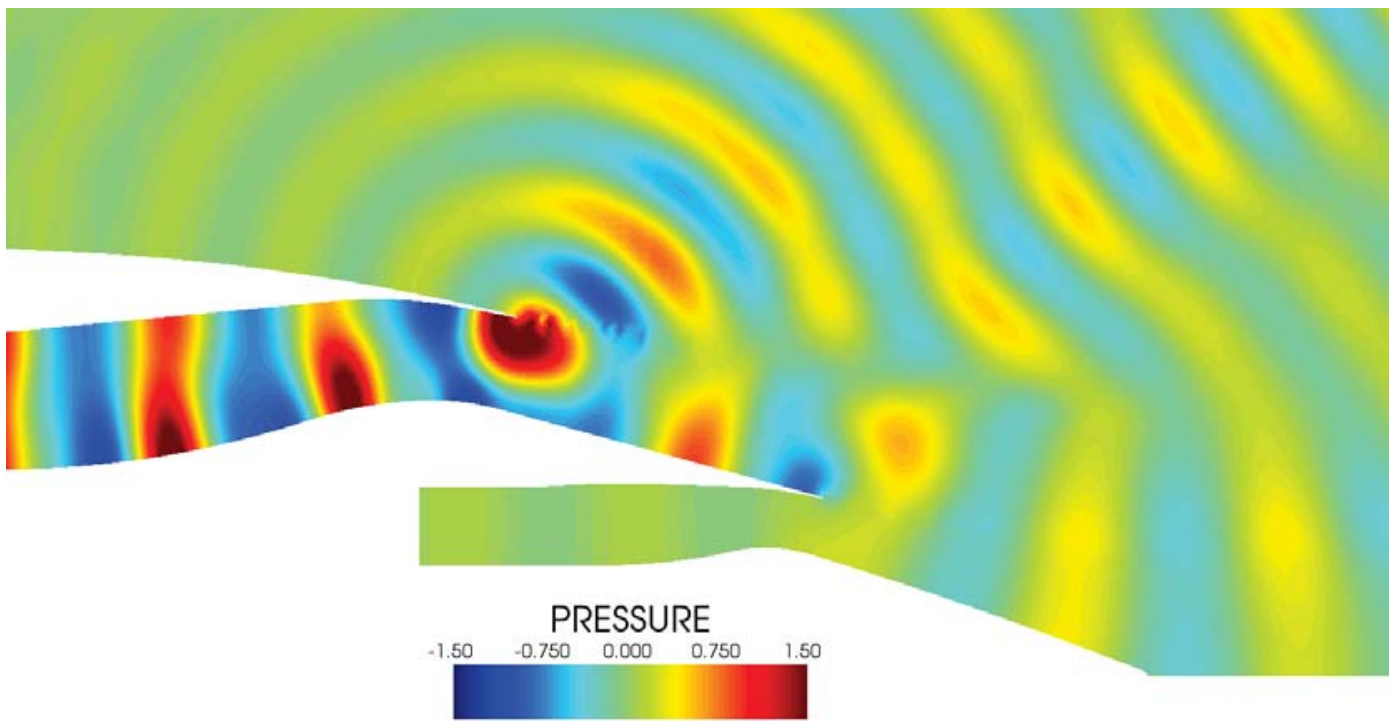

Figure 24. Pressure map for plane wave mode at frequency $7000 \mathrm{~Hz}$ 


\section{Conclusions}

- This work has allowed to set-up and validate a methodology for computing the momentum thickness of realistic shear layers.

- This methodology is applied to a realistic case in this paper. The propagation velocity and the frequency of the instabilities being consistent with the theory (see Chapter I), the instabilities observed in the ACTRAN/DGM computations are therefore physical (i.e., these are Kelvin-Helmoltz instabilities).

- The methodology described in this paper can be applied on 3D computations (see Figure 25) to confirm that the instabilities visualized are indeed the physical ones.

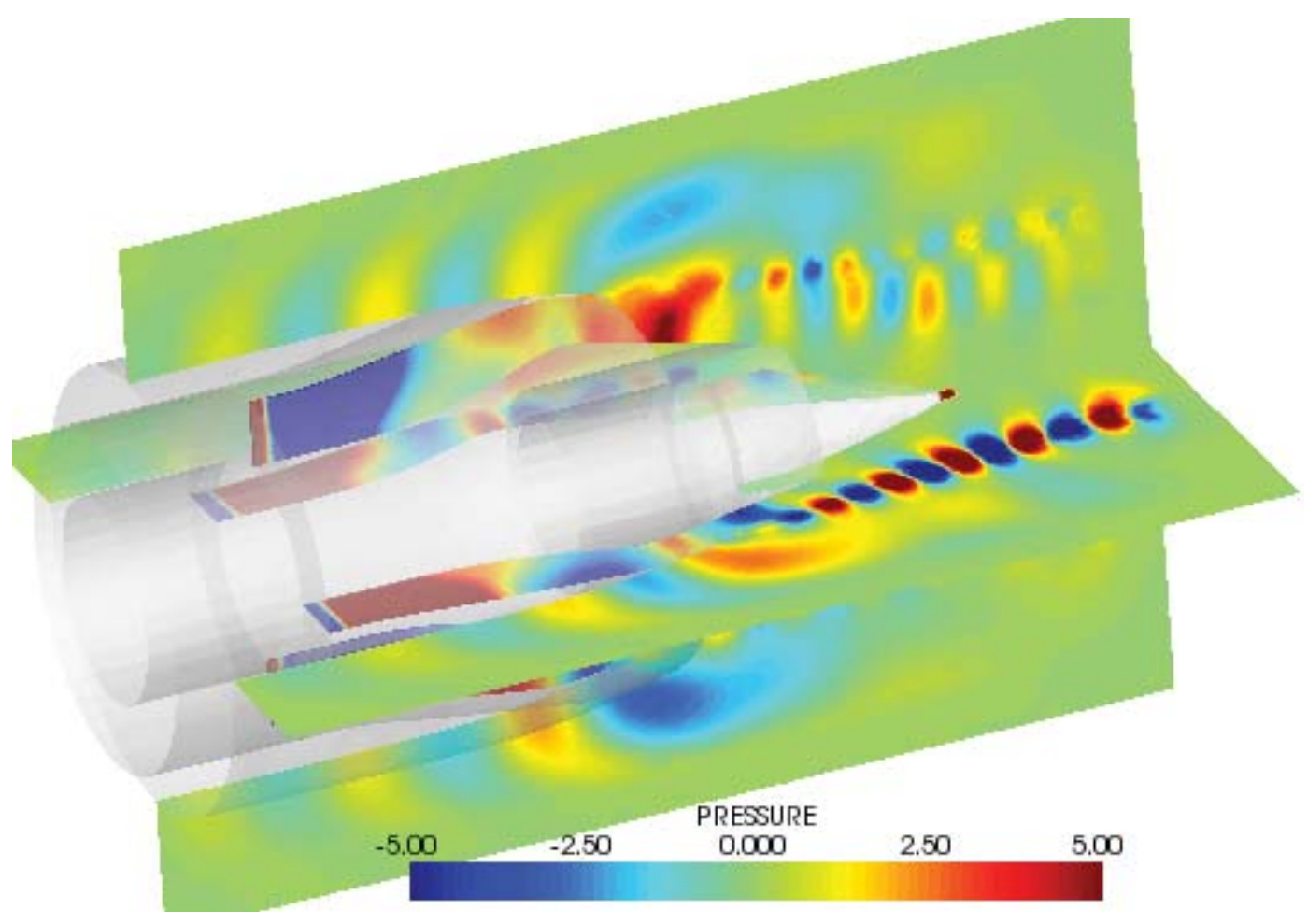

Figure 25. Pressure map for $3 \mathrm{D}$ configuration

\section{Acknowledgements}

This work has been performed within the TURNEX project, funded by the European Union under the Sixth Framework Programme (FP6), Technical Officer Daniel Chiron. The authors would like to acknowledge all the partners who have participated to this work. The authors would also like to thank Patrick Huerre for his help in understanding the phenomenon involved.

\section{References}

\footnotetext{
${ }^{1}$ Chevaugeon, N., Remacle, J., Gallez, X., Ploumhans, P., and Caro, S., "Efficient Discontinuous Galerkin Methods for solving acoustic problems," AIAA Paper 2005-2823, 11th AIAA/CEAS Aeroacoustics Conference and Exhibit, 23-25 May 2005, Monterey CA, USA.

${ }^{2}$ Chevaugeon, N., Remacle, J., and Gallez, X., "Discontinuous Galerkin Implementation of the Extended Helmholtz Resonator Impedance Model in Time Domain," AIAA Paper 2005-2560, 12th AIAA/CEAS Aeroacoustics Conference and Exhibit, May 2006, Cambridge MA, USA.

${ }^{3}$ Leneveu, R., Schiltz, B., Manera, J., and Caro, S., "Parallel DGM scheme for LEE applied to exhaust and bypass problems," AIAA Paper 2007-3510, 13th AIAA/CEAS Aeroacoustics Conference, May 2007, Rome, IT.
} 
${ }^{4}$ Kelvin, L., "Hydrokinetic solutions and observations," Philosophical Magazine, Vol. 4, No. 42, 1871, pp. 362-377.

${ }^{5}$ Helmholtz, H. v., "On discontinuous movement of fluids," Philosophical Magazine, Vol. 4, No. 36, 1868, pp. $337-346$.

${ }^{6}$ Rayleigh, L., "On the stability, or instability, of certain fluid motions," Proceedings of the London Mathematical Society, Vol. 11, 1880, pp. 57-70.

${ }^{7}$ Miles, J. W., "On the Reflection of Sound at an Interface of Relative Motion," Journal of the Acoustical Society of America, Vol. 29, No. 2, 1957, pp. 226-228.

${ }^{8}$ Crighton, D. G. and Leppington, F. G., "Radiation Properties of the Semi-Infinite Vortex Sheet: the Initial Value Problem," Journal of Fluid Mechanics, Vol. 64, No. 2, 1974, pp. 393-414.

${ }^{9}$ Morgan, J. D., "The Interaction of Sound with a Semi-Infinite Vortex Sheet," Quarterly Journal of Mechanics and Applied Mathematics, Vol. 27, No. 4, 1974, pp. 465-487.

${ }^{10}$ Munt, R. M., "The interaction of sound with a subsonic jet issuing from a semi-infinite cylindrical pipe," Journal of Fluid Mechanics, Vol. 83, 1977.

${ }^{11}$ Rienstra, S. W., "On The Acoustical Implications Of Vortex Shedding From An Exhaust Pipe," Journal of Engineering for Industry (ASME), Vol. 104, No. 4, 1981, pp. 378-384, (corr. p.112, 104, 1982).

${ }^{12}$ Rienstra, S. W., "A Small Strouhal Number Analysis For Acoustic Wave - Jet Flow - Pipe Interaction," Journal of Sound Vibration, Vol. 86, No. 4, 1983, pp. 539-556.

${ }^{13}$ Cargill, A. M., "Low frequency acoustic radiation froma jet pipe - a second order theory," Journal of Sound and Vibration, Vol. 83, 1982, pp. 339-354.

${ }^{14}$ Drazin, P. G. and Reid, W. H., Hydrodynamic Stability, Cambridge University Press, 2nd ed., 2004.

${ }^{15}$ Michalke, A., "On spatially growing disturbances in an inviscid shear layer," Journal of Fluid Mechanics, Vol. 23, No. 3, 1965, pp. 521-544.

${ }^{16}$ Michalke, A., "Survey on Jet Instability Theory," Progress in Aerospace Sciences, Vol. 21, 1984, pp. 159-199.

${ }^{17}$ Gabard, G. and Astley, R. J., "Theoretical models for sound radiation from annular jet pipes: far- and near-field solutions," Journal of Fluid Mechanics, Vol. 549, 2006, pp. 315-342.

${ }^{18}$ Demir, A. and Rienstra, S. W., "Sound Radiation from an Annular Duct with Jet Flow and a Lined Centerbody," AIAA Paper 2006-2718, 12th AIAA/CEAS Aeroacoustics Conference, Cambridge, MA, USA, 8-10 May 2006, Deliverables D2-1-1 and D2-1-2 of Turnex Project.

${ }^{19}$ Demir, A. and Rienstra, S. W., "Sound Radiation from a Buried Nozzle with Jet and Bypass Flow," paper no. 449 at ICSV14, the Fourteenth International Congress on Sound and Vibration, Cairns, July 9-12, 2007.

${ }^{20} \mathrm{Kok}$, J. C., "Computation of sound radiation from cylindrical ducts with jets using a high-order finite-volume method," AIAA Paper 2007-3489, 13th AIAA/CEAS Aeroacoustics Conference, May 2007, Rome, IT. 